\title{
Asymptotic error distribution of the Euler method for SDEs with non-Lipschitz coefficients
}

Andreas Neuenkirch and Henryk Zähle 



\title{
Asymptotic error distribution of the Euler method for SDEs with non-Lipschitz coefficients
}

\author{
Andreas Neuenkirch and Henryk Zähle
}

\begin{abstract}
In [14, 8] Kurtz and Protter resp. Jacod and Protter specify the asymptotic error distribution of the Euler method for stochastic differential equations (SDEs) with smooth coefficients growing at most linearly. The required differentiability and linear growth of the coefficients rule out some popular SDEs as for instance the Cox-Ingersoll-Ross (CIR) model, the Heston model, or the stochastic Brusselator. In this article, we partially extend one of the fundamental results in [8], so that also the mentioned examples are covered. Moreover, we compare by means of simulations the asymptotic error distributions of the CIR model and the geometric Brownian motion with mean reversion.
\end{abstract}

Keywords. Stochastic differential equation, Euler scheme, Error process, Weak convergence.

AMS classification. Primary 60H10, 65C20 Secondary 60F17.

\section{Introduction}

We consider the $d$-dimensional Itô stochastic differential equation (SDE)

$$
d X(t)=\sum_{j=0}^{m} f_{j}(X(t)) d W_{j}(t), \quad X(0)=x_{0},
$$

where $x_{0} \in \mathbb{R}^{d}, f_{0}, \ldots, f_{m}: \mathbb{R}^{d} \rightarrow \mathbb{R}^{d}$ are continuous functions, $W_{0}$ is the identity on $\mathbb{R}_{+}$(i.e. $W_{0}(t)=t$ ), and $W_{1}, \ldots, W_{m}$ are independent one-dimensional Brownian motions. SDE (1.1) and all other equations and processes in the sequel are restricted to a fixed time interval $[0, T]$. We assume that (1.1) has a unique strong solution, and we denote by $\bar{X}_{n}$ Euler's "polygonal" approximation of this solution, i.e.

$$
d \bar{X}_{n}(t)=\sum_{j=0}^{m} f_{j}\left(\bar{X}_{n}\left(\eta_{n}(t)\right)\right) d W_{j}(t), \quad \bar{X}_{n}(0)=x_{0}
$$

Here $\eta_{n}(t)$ is defined to be the largest element of $\mathbb{N}_{0} / n=\{0,1 / n, 2 / n, \ldots\}$ which is smaller than or equal to $t$. For background on the Euler scheme and other numerical schemes for SDEs see e.g. [13, 16].

This article was written while A. Neuenkirch was a member of the DFG-project "Pathwise numerics and dynamics of stochastic evolution equations" at the Johann Wolfgang Goethe-Universität Frankfurt am Main. 
In this article, we focus on the asymptotic distribution of the error process $\bar{X}_{n}-X$. It follows from results in [8] that if the functions $f_{j}$ are continuously differentiable and have at most linear growth, then the process $\sqrt{n}\left(\bar{X}_{n}-X\right)$ converges in law to the unique solution of the $d$-dimensional SDE

$$
\begin{aligned}
d U_{i}(t)= & \sum_{j=0}^{m} \nabla f_{i j}(X(t))^{\prime} U(t) d W_{j}(t) \\
& -\frac{1}{\sqrt{2}} \sum_{j=1}^{m} \sum_{l=1}^{m} \nabla f_{i j}(X(t))^{\prime} f_{l}(X(t)) d B_{l j}(t), \quad U(0)=0 .
\end{aligned}
$$

Here $\nabla f_{i j}$ is the gradient of the $i$-th component $f_{i j}$ of $f_{j}, B_{l j}(1 \leq l, j \leq m)$ are independent one-dimensional Brownian motions being independent of $W_{1}, \ldots, W_{m}$, and $v^{\prime}$ denotes the transpose of a vector $v$. The specified assumptions on the functions $f_{j}$ exclude some popular models as for instance the Cox-Ingersoll-Ross (CIR) model and the Heston model in finance, or the stochastic Brusselator used in the modeling of chemical reactions, cf. Section 3. Indeed, the mapping $x \mapsto \sqrt{|x|}$, which appears in the CIR model as well as in the Heston model, is not differentiable at 0, while the equation for the stochastic Brusselator contains polynomial coefficients. The purpose of this article is an extension of the fundamental results in $[14,8]$ to cover also the mentioned examples.

We assume that the solution of (1.1) never leaves a given open set $D \subset \mathbb{R}^{d}$, and that the functions $f_{j}$ are continuously differentiable on $D$. Our main result (Theorem 2.1) shows that under these assumptions the weak convergence of $\sqrt{n}\left(\bar{X}_{n}-X\right)$ to the solution of (1.3) still holds, where we use the convention $\nabla f_{i j}(x)=0$ for all $i=1, \ldots, d, j=0, \ldots, m$ and $x \in \mathbb{R}^{d} \backslash D$. The key for the proof is a localization procedure, similar to the one in $[5,10]$, where pathwise convergence rates for the approximation of SDEs have been derived.

Numerical results for the asymptotic error distribution are given in Section 4, where we compare the CIR model and the geometric Brownian motion.

\section{Main Result}

Let $(\Omega, \mathcal{F}, \mathbb{P})$ be a probability space, and $W=\left(W_{1}, \ldots, W_{m}\right)$ be an $m$-dimensional Brownian motion on $(\Omega, \mathcal{F}, \mathbb{P})$. As indicated in the Introduction, we assume that

(A) $\operatorname{SDE}(1.1)$ has a unique strong solution $X$ w.r.t. $\{(\Omega, \mathcal{F}, \mathbb{P}) ; W\}$.

The definition of solutions of SDEs is recalled in the Appendix A. We further assume that $X$ never leaves a given open set $D \subset \mathbb{R}^{d}$, i.e.

$$
\mathbb{P}(X(t) \in D \text { for all } t \in[0, T])=1 .
$$


Finally, we assume that

$$
\left.f_{0}\right|_{D}, \ldots,\left.f_{m}\right|_{D} \in C^{1}\left(D ; \mathbb{R}^{d}\right),
$$

where $\left.f_{j}\right|_{D}$ refers to the restriction of $f_{j}$ to $D$. For $k, l \in \mathbb{N}$ and an open set $G \subset \mathbb{R}^{k}$, we denote by $C\left(G ; \mathbb{R}^{l}\right)$ the space of all continuous functions from $G$ to $\mathbb{R}^{l}$. A superscript $p \in \mathbb{N} \cup\{\infty\}$ refers to the subclass of all $p$-times continuously differentiable functions of $C\left(G ; \mathbb{R}^{l}\right)$.

The rigorous meaning of the continuous time Euler scheme (1.2) is given by the stochastic integral scheme

$$
\bar{X}_{n}(t)=x_{0}+\sum_{j=0}^{m} \int_{0}^{t} f_{j}\left(\bar{X}_{n}\left(\eta_{n}(s)\right)\right) d W_{j}(s) .
$$

We now turn to the main result, whose proof will be carried out in Section 5 . We equip $C\left([0, T] ; \mathbb{R}^{m} \otimes \mathbb{R}^{d}\right)$ with the supremum norm, and we use the symbol " $\Longrightarrow$ " for weak convergence.

Theorem 2.1. Let $X$ and $f_{0}, \ldots, f_{m}$ satisfy assumptions $(A),(B)$ and $(C)$. Then there are an extension $(\bar{\Omega}, \overline{\mathcal{F}}, \overline{\mathbb{P}})$ of the original probability space (i.e. of the domain of $\left.W_{1}, \ldots, W_{m}\right)$ and independent Brownian motions $B_{l j}(1 \leq l, j \leq m)$ on $(\bar{\Omega}, \overline{\mathcal{F}}, \overline{\mathbb{P}})$ which are independent of $W_{1}, \ldots, W_{m}$, such that $S D E(1.3)$ has a unique solution $U$ (w.r.t. $\left.(\bar{\Omega}, \overline{\mathcal{F}}, \overline{\mathbb{P}}),\left\{W_{j}\right\},\left\{B_{l j}\right\}\right)$, and we have

$$
\left(W, \sqrt{n}\left(\bar{X}_{n}-X\right)\right) \Longrightarrow(W, U) \quad\left(\text { in } C\left([0, T] ; \mathbb{R}^{m} \otimes \mathbb{R}^{d}\right)\right) .
$$

The continuous time Euler scheme $\bar{X}_{n}$ is indeed not an implementable approximation scheme since it requires complete knowledge of the sample paths of the driving Brownian motions. However, in practice one is often only interested in the values of $\bar{X}_{n}$ at the sampling points $0,1 / n, 2 / n, \ldots$, i.e. in the discrete time Euler scheme. For the latter we can derive from Theorem 2.1 the following weak convergence result:

Corollary 2.2. Let $X$ and $f_{0}, \ldots, f_{m}$ satisfy assumptions $(A),(B)$ and $(C)$. Then

$$
\sqrt{n} \max _{i=0, \ldots, N_{n}(T)}\left|\bar{X}_{n}(i / n)-X(i / n)\right| \Longrightarrow \max _{t \in[0, T]}|U(t)|,
$$

where $N_{n}(T)=\max \{i \in \mathbb{N}: i / n \leq T\}$.

Proof. Theorem 2.1 implies in particular weak convergence of $\sqrt{n}\left(\bar{X}_{n}-X\right)$ to $U$ in the space $\mathbb{D}=\mathbb{D}\left([0, T] ; \mathbb{R}^{d}\right)$ of cádlàg functions (i.e. functions that are rightcontinuous and have left limits) equipped with the Skorohod metric. Moreover, the function $\eta_{n}$ introduced subsequent to (1.2) converges to the identity on $[0, T]$. Therefore Lemma 2.2 of [8] yields weak convergence of $\sqrt{n}\left(\bar{X}_{n}-X\right) \circ \eta_{n}$ to $U$ in $\mathbb{D}$. Since the limit process $U$ is continuous, the latter convergence also holds if $\mathbb{D}$ is equipped 
with the supremum norm, cf. [18, p.137]. Thus, as the mapping $\phi \mapsto \max _{t \in[0, T]}|\phi(t)|$ from $\mathbb{D}$ to $\mathbb{R}$ is continuous with respect to the supremum norm, the continuous mapping theorem shows that

$$
\begin{aligned}
& \sqrt{n} \max _{i=0, \ldots, N_{n}(T)}\left|\bar{X}_{n}(i / n)-X(i / n)\right| \\
& =\sqrt{n} \max _{t \in[0, T]}\left|\bar{X}_{n}\left(\eta_{n}(t)\right)-X\left(\eta_{n}(t)\right)\right|
\end{aligned}
$$

converges weakly to the right-hand side of (2.2).

If $D \neq \mathbb{R}^{d}$ then, in contrast to the exact solution, the Euler scheme may leave $D$. For many purposes this is an unwanted property. To overcome this problem one can consider a projected Euler scheme with $\bar{X}_{n}^{(\pi)}(0)=x_{0}$ and

$$
\bar{X}_{n}^{(\pi)}((i+1) / n)=H(i / n) \mathbb{1}_{D}(H(i / n))+\pi(H(i / n)) \mathbb{1}_{\mathbb{R}^{d} \backslash D}(H(i / n))
$$

for $i=0,1, \ldots$, where

$$
H(i / n)=\bar{X}_{n}^{(\pi)}(i / n)+\sum_{j=0}^{m} f_{j}\left(\bar{X}_{n}^{(\pi)}(i / n)\right)\left(W_{j}((i+1) / n)-W_{j}(i / n)\right)
$$

and $\pi: \mathbb{R}^{d} \rightarrow D$. Such projected Euler methods have been originally introduced for the approximation of SDEs with reflecting boundaries, see e.g. [15, 17]. Of course, the reflection function $\pi$ should be chosen appropriately, i.e. according to the structure of the SDE. However, we obtain the analogue of Corollary 2.2 also for the projected Euler scheme regardless of the choice of $\pi$ :

Corollary 2.3. Let $X$ and $f_{0}, \ldots, f_{m}$ satisfy assumptions $(A),(B)$ and $(C)$, let $N_{n}(T)$ be as in Corollary 2.2, and let $\pi: \mathbb{R}^{d} \rightarrow D$ be an arbitrary function. Then

$$
\sqrt{n} \max _{i=0, \ldots, N_{n}(T)}\left|\bar{X}_{n}^{(\pi)}(i / n)-X(i / n)\right| \Longrightarrow \max _{t \in[0, T]}|U(t)| .
$$

Proof. In the proof of Lemma 5.3 below it is implicitly shown that for $\mathbb{P}$-almost all $\omega \in \Omega$ there exists some $n_{0}(\omega) \in \mathbb{N}$, such that $\bar{X}_{n}(., \omega)$ does not leave $D$ for all $n \in \mathbb{N}$ with $n \geq n_{0}(\omega)$. Thus, since $\bar{X}_{n}^{(\pi)}(., \omega)$ coincides with $\bar{X}_{n}(., \omega)$ as long as the latter takes values only in $D$, we have $\bar{X}_{n}^{(\pi)}(., \omega)=\bar{X}_{n}(., \omega)$ for all $n \in \mathbb{N}$ with $n \geq n_{0}(\omega)$. In particular, we obtain $\left(M_{n}-M_{n}^{(\pi)}\right) \rightarrow 0$ as $n \rightarrow \infty \mathbb{P}$-almost surely, where

$$
\begin{aligned}
M_{n} & =\sqrt{n} \max _{i=0, \ldots, N_{n}(T)}\left|\bar{X}_{n}(i / n)-X(i / n)\right|, \\
M_{n}^{(\pi)} & =\sqrt{n} \max _{i=0, \ldots, N_{n}(T)}\left|\bar{X}_{n}^{(\pi)}(i / n)-X(i / n)\right|,
\end{aligned}
$$


and so $M_{n}^{(\pi)}=M_{n}+\left(M_{n}^{(\pi)}-M_{n}\right)$ converges weakly to $\max _{t \in[0, T]}|U(t)|$ as $n \rightarrow \infty$ by Corollary 2.2 and Slutzky’s lemma.

\section{Examples}

In this section, we illustrate our main result by means of four examples. The first example (Subsection 3.1) is also covered by the fundamental results in $[14,8]$, but the other three examples are not.

\subsection{Geometric Brownian motion}

The geometric Brownian motion with mean reversion, which is given by the unique solution of the one-dimensional SDE

$$
d A(t)=\kappa(\lambda-A(t)) d t+\theta A(t) d W(t), \quad A(0)=a_{0}>0
$$

with $\kappa, \lambda, \theta>0$, is a popular model for the dynamics of asset prices. Note that the solution of (3.1) remains strictly positive for all time, so that the process is indeed suitable to model the dynamics of asset prices. Clearly, Theorem 2.1 applies here for $D=\mathbb{R}$ and we have

$$
d U(t)=-\kappa U(t) d t+\theta U(t) d W(t)-\frac{\theta^{2}}{\sqrt{2}} A(t) d B_{11}(t), \quad U(0)=0,
$$

which gives

$$
U(t)=-\frac{\theta^{2}}{\sqrt{2}} \Phi(t) \int_{0}^{t} \frac{1}{\Phi(s)} A(s) d B_{11}(s),
$$

where $\Phi$ is the unique solution of the one-dimensional linear SDE

$$
d \Phi(t)=-\kappa \Phi(t) d t+\theta \Phi(t) d W(t), \quad \Phi(0)=1,
$$

see (5.3) in Subsection 5.1. Of course, the solution of the latter SDE is given by

$$
\Phi(t)=\exp \left(-\left(\kappa+\frac{1}{2} \theta^{2}\right) t+\theta W(t)\right) .
$$

Since the solution of (3.1) has the representation

$$
A(t)=\Phi(t)\left(a_{0}+\kappa \lambda \int_{0}^{t} \frac{1}{\Phi(s)} d s\right)
$$

the expression for (3.2) can be rewritten as

$$
U(t)=-\frac{\theta^{2}}{\sqrt{2}} \Phi(t)\left(a_{0} B_{11}(t)+\kappa \lambda \int_{0}^{t} \int_{0}^{s} \frac{1}{\Phi(u)} d u d B_{11}(s)\right) .
$$




\subsection{Cox-Ingersoll-Ross model}

The Cox-Ingersoll-Ross (CIR) process is given by the unique solution of the onedimensional SDE

$$
d V(t)=\kappa(\lambda-V(t)) d t+\theta \sqrt{|V(t)|} d W(t), \quad V(0)=v_{0}>0
$$

with $\kappa, \lambda, \theta>0$. It is well known that if $2 \kappa \lambda \geq \theta^{2}$, then the solution remains strictly positive for all time (cf., e.g., [21, Section 3]). Since this is a desired property for interest rates, Cox et al. ([3]) proposed this process in 1985 as a model for short-term interest rates. A further advantage of the CIR process in the context of short rates is that it admits closed-form formulae for bond prices. The strict positivity of the solution ensures that Theorem 2.1 applies for $D=(0, \infty)$ and we have

$$
d U(t)=-\kappa U(t) d t+\frac{\theta}{2} \frac{1}{\sqrt{V(t)}} U(t) d W(t)-\frac{\theta^{2}}{\sqrt{8}} d B_{11}(t), \quad U(0)=0,
$$

which gives

$$
U(t)=-\frac{\theta^{2}}{\sqrt{8}} \Phi(t) \int_{0}^{t} \frac{1}{\Phi(s)} d B_{11}(s),
$$

where $\Phi$ is the unique solution of the one-dimensional linear SDE

$$
d \Phi(t)=-\kappa \Phi(t) d t+\frac{\theta}{2} \frac{1}{\sqrt{V(t)}} \Phi(t) d W(t), \quad \Phi(0)=1,
$$

see again (5.3) in Subsection 5.1. The solution of the latter SDE reads as

$$
\Phi(t)=\exp \left(-\kappa t-\frac{\theta^{2}}{8} \int_{0}^{t} \frac{1}{V(s)} d s+\frac{\theta}{2} \int_{0}^{t} \frac{1}{\sqrt{V(s)}} d W(s)\right) .
$$

Note that if the condition $2 \kappa \lambda \geq \theta^{2}$ is violated, then the solution of (3.3) can still be approximated weakly by the Euler scheme (cf. [20, Section 4]) but it may obtain the value zero. (It will even obtain the value zero with probability one if the time horizon is infinite, cf. [21, Section 3]). Thus in this case our results do not apply.

\subsection{Heston model}

A popular stochastic volatility model in finance is the Heston model ([6]), i.e.

$$
\begin{aligned}
& d A(t)=\mu A(t) d t+A(t)\left[\rho \sqrt{|V(t)|} d W_{1}(t)+\sqrt{1-\rho^{2}} \sqrt{|V(t)|} d W_{2}(t)\right], \\
& d V(t)=\kappa(\lambda-V(t)) d t+\theta \sqrt{|V(t)|} d W_{1}(t),
\end{aligned}
$$


where $\kappa, \lambda, \theta, \mu>0$ and $\rho \in[-1,1]$. Here, a process $(V(t): t \in[0, T])$ of CoxIngersoll-Ross type is used to model the volatility of an asset $(A(t): t \in[0, T])$. If $2 \kappa \lambda \geq \theta^{2}, V(0)>0$ and $A(0)>0$, then the unique solution of the two-dimensional $\operatorname{SDE}$ (3.5) remains in $D=(0, \infty)^{2}$. Therefore Theorem 2.1 applies and we have the following asymptotic dynamics of the error process:

$$
\begin{aligned}
{\left[\begin{array}{l}
d U_{1}(t) \\
d U_{2}(t)
\end{array}\right]=} & {\left[\begin{array}{r}
\mu U_{1}(t) \\
-\kappa U_{2}(t)
\end{array}\right] d t } \\
+ & {\left[\begin{array}{c}
\rho \sqrt{V(t)} U_{1}(t)+\frac{\rho}{2} \frac{A(t)}{\sqrt{V(t)}} U_{2}(t) \\
\frac{\theta}{2} \frac{1}{\sqrt{V(t)}} U_{2}(t)
\end{array}\right] d W_{1}(t) } \\
+ & {\left[\begin{array}{c}
\sqrt{1-\rho^{2}} \sqrt{V(t)} U_{1}(t)+\frac{\sqrt{1-\rho^{2}}}{2} \frac{A(t)}{\sqrt{V(t)}} U_{2}(t) \\
0
\end{array}\right] d W_{2}(t) } \\
+ & {\left[\begin{array}{c}
d R_{1}(t) \\
d R_{2}(t)
\end{array}\right] }
\end{aligned}
$$

where

$$
\begin{aligned}
{\left[\begin{array}{l}
d R_{1}(t) \\
d R_{2}(t)
\end{array}\right]=} & -\frac{1}{\sqrt{2}}\left[\begin{array}{c}
\rho^{2} A(t) V(t)+\frac{\rho \theta}{2} A(t) \\
\frac{\theta^{2}}{2}
\end{array}\right] d B_{11}(t) \\
& -\frac{1}{\sqrt{2}}\left[\begin{array}{c}
\rho \sqrt{1-\rho^{2}} A(t) V(t) \\
0
\end{array}\right] d B_{21}(t) \\
& -\frac{1}{\sqrt{2}}\left[\begin{array}{c}
\rho \sqrt{1-\rho^{2}} A(t) V(t)+\frac{\theta \sqrt{1-\rho^{2}}}{2} A(t) \\
0
\end{array}\right] d B_{12}(t) \\
& -\frac{1}{\sqrt{2}}\left[\begin{array}{c}
\left(1-\rho^{2}\right) A(t) V(t) \\
0
\end{array}\right] d B_{22}(t) .
\end{aligned}
$$

\subsection{Stochastic Brusselator}

An example for an SDE with polynomial coefficients is the stochastic Brusselator

$$
\begin{aligned}
& d X(t)=\left(\alpha-(\beta+1) X(t)+X(t)^{2} Y(t)+\frac{\sigma^{2}}{2} X(t)\right) d t-\sigma X(t) d W(t), \\
& d Y(t)=\left(\beta X(t)-X(t)^{2} Y(t)-\frac{\sigma^{2}}{2} X(t)\right) d t+\sigma X(t) d W(t),
\end{aligned}
$$

where $\alpha, \beta, \sigma>0$. Although the coefficients of this SDE have polynomial growth, this equation admits a unique strong solution, see e.g. [19, 2]. Here Theorem 2.1 for 


$$
\begin{aligned}
& D=\mathbb{R}^{2} \text { gives } \\
& \left.\qquad \begin{array}{l}
d U_{1}(t) \\
d U_{2}(t)
\end{array}\right]= \\
& +\left[\begin{array}{r}
-\sigma U_{1}(t) \\
\sigma U_{1}(t)
\end{array}\right] d W(t)-\frac{1}{\sqrt{2}}\left[\begin{array}{r}
\sigma^{2} X(t) \\
-\sigma^{2} X(t)
\end{array}\right] d B_{11}(t) .
\end{aligned}
$$

\section{Numerical Examples}

Here we compare the asymptotic error processes of the geometric Brownian motion with mean reversion (3.1) and the CIR model (3.3). We set $T=1$ and focus on the distribution of the random variable

$$
M=\max _{t \in[0,1]}|U(t)| .
$$

We consider two different settings:

1) For the CIR process $V=(V(t): t \in[0,1])$ we choose

$$
\kappa=0.97351, \quad \lambda=0.05791, \quad \theta=0.15415, \quad \text { and } \quad v_{0}=0.02 \text {, }
$$

which corresponds to the dynamics of the short term interest rates in the CIR model (under the objective measure) estimated from historical data of the German debt security market (Rentenmarkt), cf. [4]. For the geometric Brownian motion $A=(A(t)$ : $t \in[0,1])$ the parameters are chosen in such a way that $A(1)$ and $V(1)$ have the same variance:

$$
\kappa=0.97351, \quad \lambda=0.05791, \quad \theta=0.3988, \quad \text { and } \quad a_{0}=0.02 .
$$

2) For the CIR process $V=(V(t): t \in[0,1])$ we choose

$$
\kappa=1, \quad \lambda=0.5, \quad \theta=1, \quad \text { and } \quad v_{0}=0.2,
$$

so that the strict positivity condition $2 \kappa \lambda \geq \theta^{2}$ is just satisfied. For the geometric Brownian motion $A=(A(t): t \in[0,1])$ the parameters are again chosen in such a way that $A(1)$ and $V(1)$ have the same variance:

$$
\kappa=1, \quad \lambda=0.5, \quad \theta=1.2944, \quad \text { and } \quad a_{0}=0.2 .
$$

For both settings 1) and 2) we simulated 10.000 paths of $U$ for both $A$ and $V$, where we used the representations (3.2) and (3.4). Figure 1 shows the empirical densities of $M$ in either case. We used a kernel density estimator with Gaussian kernel and bandwidth choice by cross-validation. The following table displays some further empirical features of $M$ : 


\begin{tabular}{crrrrrr}
\hline setting & model & mean & sd & min & max & kurt \\
\hline 1$)$ & GBM & 0.015643 & 0.009530 & 0.003225 & 0.162262 & 19.76 \\
& CIR & 0.008937 & 0.003206 & 0.003157 & 0.028918 & 4.64 \\
\hline 2$)$ & GBM & 0.548598 & 0.613945 & 0.055889 & 15.861450 & 119.69 \\
& CIR & 0.395864 & 0.163062 & 0.129266 & 1.653468 & 6.22 \\
\hline
\end{tabular}
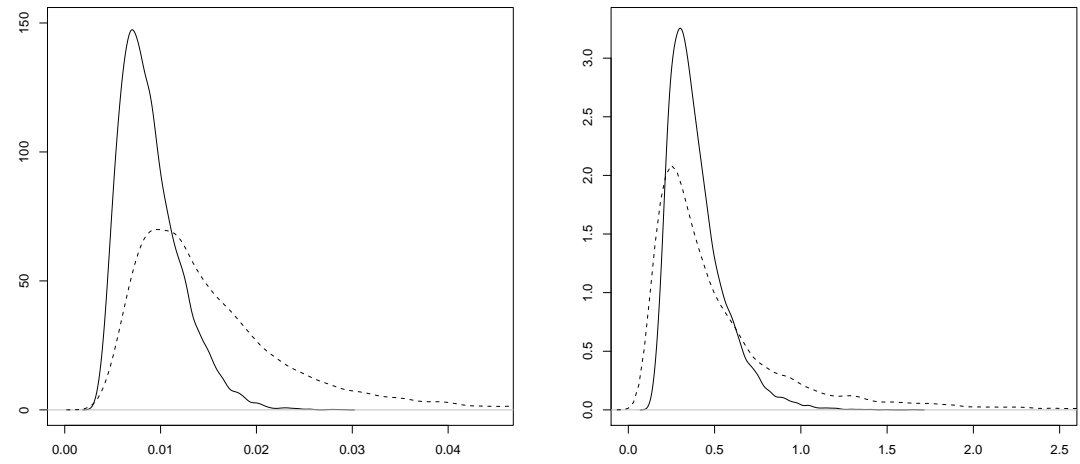

Figure 1. Empirical density of $M$ for the CIR model and for the geometric Brownian motion (dashed line). Left: setting 1), right: setting 2).

Surprisingly, in both cases the empirical densities for the geometric Brownian motion are broader than the densities for the CIR process. (See also the corresponding means and variances in the above table.) In particular, the non-Lipschitz coefficient in SDE (3.3) does not lead to a badly shaped asymptotic error distribution as one might expect.

\section{Proof of Theorem 2.1}

The proof of Theorem 2.1 is organized as follows. First, in Subsection 5.1 we discuss the existence and uniqueness of solutions to equation (1.3). Second, in Subsections 5.2 and 5.3 we carry out the proof of (2.1). Finally, in Subsections 5.4 and 5.5 we give the proofs of two lemmas, which are omitted in Subsection 5.2.

\subsection{Unique solution of (1.3)}

Note that equation (1.3) can be rewritten as

$$
d U(t)=d H(t)+d S(t) U(t)
$$


where $H$ and $S$ are $\mathbb{R}^{d}$ - resp. $\mathbb{R}^{d, d}$-valued continuous semi-martingales:

$$
\begin{gathered}
H(t)=\left[\begin{array}{c}
-\frac{1}{\sqrt{2}} \sum_{j=1}^{m} \sum_{l=1}^{m} \int_{0}^{t} \nabla f_{1 j}(X(s))^{\prime} f_{l}(X(s)) d B_{l j}(s) \\
\vdots \\
-\frac{1}{\sqrt{2}} \sum_{j=1}^{m} \sum_{l=1}^{m} \int_{0}^{t} \nabla f_{d j}(X(s))^{\prime} f_{l}(X(s)) d B_{l j}(s)
\end{array}\right], \\
S(t)=\sum_{j=0}^{m}\left[\begin{array}{ccc}
\int_{0}^{t} \partial_{1} f_{1 j}(X(s)) d W_{j}(s) & \cdots & \int_{0}^{t} \partial_{d} f_{1 j}(X(s)) d W_{j}(s) \\
\vdots & & \vdots \\
\int_{0}^{t} \partial_{1} f_{d j}(X(s)) d W_{j}(s) & \cdots & \int_{0}^{t} \partial_{d} f_{d j}(X(s)) d W_{j}(s)
\end{array}\right] .
\end{gathered}
$$

Here $\partial_{k} f_{i j}$ denotes the $k$-th partial derivative of $f_{i j}$. For the precise meaning of equation (5.1) see Definition A.2 in the Appendix A. Since the Brownian motions $W_{1}, \ldots, W_{m}$ and $B_{l j}(1 \leq j, l \leq m)$ are independent, the covariation of $H$ and $S$ is zero. Now it follows from Lemma A.3 in the Appendix A that equation (5.1) (and thus (1.3)) has a unique solution which is given by

$$
U(t)=\Phi(t) \int_{0}^{t} \Phi(s)^{-1} d H(s) .
$$

Here, $(\Phi(t): t \in[0, T])$ is the unique solution of the $\mathbb{R}^{d, d}$-valued linear SDE

$$
d \Phi(t)=d S(s) \Phi(t), \quad \Phi(0)=\mathbb{I}_{d},
$$

where $\Phi(s)^{-1}$ denotes the inverse of the matrix $\Phi(s)$.

\subsection{Proof of (2.1) (Step 1)}

We now turn to the proof of (2.1), which avails a localization procedure similar to that of $[5,10]$. We start with introducing a truncated version of SDE (1.1). For every $q \in \mathbb{N}$, we set

$$
D_{q}=\{x \in D:|x|<q \text { and } \operatorname{dist}(x, \partial D)>1 / q\}
$$

with $\partial D$ the boundary of $D$, and $\operatorname{dist}(x, \partial D)=\inf \{|x-y|: y \in \partial D\}$. Moreover, denote $q_{0}=\min \left\{q \in \mathbb{N}: x_{0} \in D_{q}\right\}$. In particular, we then have $\cup_{q \geq q_{0}} D_{q}=D$. For every $q \in \mathbb{N}$ there exists a function $\varphi_{q} \in C^{\infty}\left(\mathbb{R}^{d} ; \mathbb{R}\right)$ such that $0 \leq \varphi_{q}(x) \leq 1$ and

$$
\varphi_{q}(x)= \begin{cases}1 & , \quad x \in \bar{D}_{q}, \\ 0 & , \quad x \in \mathbb{R}^{d} \backslash D_{2 q} .\end{cases}
$$


We use these functions to truncate the coefficients of SDE (1.1), i.e. we set

$$
f_{j, q}(x)=f_{j}(x) \varphi_{q}(x), \quad x \in \mathbb{R}^{d},
$$

for all $j=0, \ldots, m$ and $q \in \mathbb{N}$. The corresponding SDE reads as follows

$$
d X^{(q)}(t)=\sum_{j=0}^{m} f_{j, q}\left(X^{(q)}(t)\right) d W_{j}(t), \quad X^{(q)}(0)=x_{0} .
$$

Clearly, we have $f_{j, q} \in C^{1}\left(\mathbb{R}^{d} ; \mathbb{R}^{d}\right)$ and the derivative of $f_{j, q}$ is bounded. Therefore the functions $f_{j, q}$ are Lipschitz continuous, so the standard theory ensures that the truncated SDE (5.5) has a unique strong solution $X^{(q)}$. On the other hand, the truncated coefficients also satisfy the assumptions of the following theorem.

Theorem 5.1. Suppose that SDE (1.1) has a unique strong solution, and that the functions $f_{0}, \ldots, f_{m}$ are continuously differentiable on $\mathbb{R}^{d}$ and have at most linear growth (i.e. $\left.\left|f_{j}(x)\right| \leq K(1+|x|)\right)$. Further, define the processes $Z_{n}=\left(Z_{n}^{i, j}, 1 \leq i, j \leq m\right)$ and $U_{n}$ by

$$
\begin{gathered}
Z_{n}^{i, j}(t):=\sqrt{n} \int_{0}^{t}\left(W_{i}(s)-W_{i}(\eta(s))\right) d W_{j}(s), \\
U_{n}:=\sqrt{n}\left(\bar{X}_{n}-X\right) .
\end{gathered}
$$

Then there are an extension $(\bar{\Omega}, \overline{\mathcal{F}}, \overline{\mathbb{P}})$ of the original probability space (i.e. of the domain of $\left.W_{1}, \ldots, W_{m}\right)$ and independent Brownian motions $B_{l j}(1 \leq l, j \leq m)$ on $(\bar{\Omega}, \overline{\mathcal{F}}, \overline{\mathbb{P}})$ which are independent of $W_{1}, \ldots, W_{m}$, such that $S D E(1.3)$ has a unique solution $U$ (w.r.t. $\left.(\bar{\Omega}, \overline{\mathcal{F}}, \overline{\mathbb{P}}),\left\{W_{j}\right\},\left\{B_{l j}\right\}\right)$ and

$$
\left(W, Z_{n}, U_{n}\right) \Longrightarrow(W, B, U) \quad\left(\text { in } C\left([0, T] ; \mathbb{R}^{m} \otimes \mathbb{R}^{m, m} \otimes \mathbb{R}^{d}\right)\right) .
$$

Here the extension $(\bar{\Omega}, \overline{\mathcal{F}}, \overline{\mathbb{P}})$ may be chosen to be independent of $f_{0}, \ldots, f_{m}$.

Proof. For the existence and uniqueness of a strong solution of SDE (1.3) see Subsection 5.1. The other claims are implied by Theorem 3.2 of [8] and its proof (along with the implication (a) $\Rightarrow$ (b) in Theorem 5.5 of [8]). Actually, the results in [8] only give the weak convergence in (5.6) in the cádlàg space $\mathbb{D}\left([0, T] ; \mathbb{R}^{m} \otimes \mathbb{R}^{m, m} \otimes \mathbb{R}^{d}\right)$. However, since the limit $(W, B, U)$ is continuous, the weak convergence also holds in $C\left([0, T] ; \mathbb{R}^{m} \otimes \mathbb{R}^{m, m} \otimes \mathbb{R}^{d}\right)$.

Therefore we obtain for $U_{n}^{(q)}:=\sqrt{n}\left(\bar{X}_{n}^{(q)}-X^{(q)}\right), Z_{n}$ and $\left\{B_{l j}\right\}$ as in Theorem 5.1 that

$$
\left(W, Z_{n}, U_{n}^{(q)}\right) \Longrightarrow\left(W, B, U^{(q)}\right),
$$

where $\bar{X}_{n}^{(q)}$ is the continuous time Euler scheme for the approximation of SDE (5.5), and $U^{(q)}$ is the unique strong solution of $\operatorname{SDE}(1.3)$ with $X$ and $f_{j}$ replaced by $X^{(q)}$ and 
$f_{j, q}$, respectively. Note that we may and do assume that all limits $\left(W, B, U^{(q)}\right), q \in \mathbb{N}$, are defined on the same extension $(\bar{\Omega}, \overline{\mathcal{F}}, \overline{\mathbb{P}})$ of the original domain, since the extension $(\bar{\Omega}, \overline{\mathcal{F}}, \overline{\mathbb{P}})$ in Theorem 5.1 depends only on $B$ (i.e. is independent of $q$ ). Further, we denote by $U$ the unique strong solution of (1.3) with respect to $\left((\bar{\Omega}, \overline{\mathcal{F}}, \overline{\mathbb{P}}),\left\{W_{j}\right\},\left\{B_{l j}\right\}\right)$.

Now, our objective is to derive (2.1) by means of (5.7). This will be done in Subsection 5.3, for which we need some further preparation. We define

$$
\tau_{q}=\inf \left\{t \in[0, T]: X(t) \notin D_{q}\right\}
$$

and

$$
\tau_{q}^{(q)}=\inf \left\{t \in[0, T]: X^{(q)}(t) \notin D_{q}\right\},
$$

for every $q \in \mathbb{N}$, where we use the convention $\inf \emptyset=\infty$. The stopping times $\tau_{q}$ and $\tau_{q}^{(q)}$ specify the first exit times of $X$ and $X^{(q)}$ from the set $D_{q}$. Note that we have

$$
\lim _{q \rightarrow \infty} \tau_{q}=\infty,
$$

since $X$ is continuous and never leaves $D$. For the considerations of Subsection 5.3 the following three lemmas are crucial. The first one states that $\tau_{q}$ and $\tau_{q}^{(q)}$ coincide, and that $X$ and $X^{(q)}$ coincide up to this exit time. The second one shows that the two Euler schemes $\bar{X}_{n}$ and $\bar{X}_{n}^{(q)}$ coincide on the set $\left\{\tau_{q}=\infty\right\}$ for $n$ sufficiently large, and the last one says that the processes $U$ and $U^{(q)}$ coincide also up to the first exit time $\tau_{q}$. The first lemma is more or less obvious. Therefore we omit its proof. The proofs of the other two lemmas are postponed to Subsections 5.4 and 5.5.

Lemma 5.2. Let $q \geq q_{0}$. We have $\mathbb{P}$-almost surely, $X\left(. \wedge \tau_{q}\right)=X^{(q)}\left(. \wedge \tau_{q}\right)$ and $\tau_{q}=\tau_{q}^{(q)}$.

Lemma 5.3. Let $q \geq q_{0}$. For $\mathbb{P}$-almost all $\omega \in \Omega$ there exists some $n_{0}(\omega) \in \mathbb{N}$ such that for all $n \in \mathbb{N}$ with $n \geq n_{0}(\omega)$,

$$
\bar{X}_{n}(., \omega) \mathbb{1}_{\left\{\tau_{q}(\omega)=\infty\right\}}(\omega)=\bar{X}_{n}^{(q)}(., \omega) \mathbb{1}_{\left\{\tau_{q}(\omega)=\infty\right\}}(\omega) .
$$

Lemma 5.4. Let $q \geq q_{0}$. We have $\overline{\mathbb{P}}$-almost surely, $U\left(. \wedge \tau_{q}\right)=U^{(q)}\left(. \wedge \tau_{q}\right)$.

\subsection{Proof of (2.1) (Step 2)}

In order to prove (2.1), we have to show

$$
\lim _{n \rightarrow \infty} \mathbb{E}\left[g\left(W, U_{n}\right)\right]=\overline{\mathbb{E}}[g(W, U)]
$$


for all bounded and continuous functions $g: C\left([0, T] ; \mathbb{R}^{m} \otimes \mathbb{R}^{d}\right) \rightarrow \mathbb{R}$. Since we have $\overline{\mathbb{P}} \equiv \mathbb{P}$ on $(\Omega, \mathcal{F}, \mathbb{P})$, we will always write $\overline{\mathbb{E}}$ instead of $\mathbb{E}$ in this subsection. For every $q \geq q_{0}+1$, let

$$
A_{q}=\left\{\tau_{q-1} \leq T\right\} \cap\left\{\tau_{q}=\infty\right\}
$$

be the event that $X$ leaves $D_{q-1}$ and never leaves $D_{q}$. Moreover, set $A_{q_{0}}=\left\{\tau_{q_{0}}=\right.$ $\infty\}$. With the help of (5.8), Lemma 5.2, the dominated convergence theorem (recall that $g$ is bounded), and by introducing a telescoping sum, we obtain

$$
\begin{aligned}
& \overline{\mathbb{E}} {\left[g\left(W, U_{n}\right)\right] } \\
&= \overline{\mathbb{E}}\left[\sum_{q=q_{0}}^{\infty} g\left(W, \sqrt{n}\left(\bar{X}_{n}-X\right)\right) \mathbb{1}_{A_{q}}\right] \\
&= \sum_{q=q_{0}}^{\infty} \overline{\mathbb{E}}\left[g\left(W, \sqrt{n}\left(\bar{X}_{n}-X^{(q)}\right)\right) \mathbb{1}_{A_{q}}\right] \\
&= \sum_{q=q_{0}}^{\infty}\left(\overline{\mathbb{E}}\left[g\left(W, \sqrt{n}\left(\bar{X}_{n}^{(q)}-X^{(q)}\right)\right) \mathbb{1}_{A_{q}}\right]\right. \\
&\left.\quad+\overline{\mathbb{E}}\left[\left\{g\left(W, \sqrt{n}\left(\bar{X}_{n}-X^{(q)}\right)\right)-g\left(W, \sqrt{n}\left(\bar{X}_{n}^{(q)}-X^{(q)}\right)\right)\right\} \mathbb{1}_{A_{q}}\right]\right) \\
&=: \quad \sum_{q=q_{0}}^{\infty}\left(S_{1}(n, q)+S_{2}(n, q)\right) .
\end{aligned}
$$

Now note that $\mathbb{1}_{A_{q}}$ is measurable with respect to $\mathcal{W}:=\sigma(W(s): s \in[0, T])$. Thus, by the factorization lemma there exists a $\mathcal{W}$-measurable (and bounded) function $F_{q}$ : $C\left([0, T] ; \mathbb{R}^{d}\right) \rightarrow \mathbb{R}$ such that $\mathbb{1}_{A_{q}}=F_{q}(W)$. Moreover, the bounded and continuous functions are dense in $L^{2}\left(C, \mathcal{C}, \underline{\mathbb{P}} \circ W^{-1}\right)$ (cf. e.g. [11, Lemma 1.33]), where $C=$ $C\left([0, T] ; \mathbb{R}^{d}\right)$ and $\mathcal{C}$ is the Borel $\sigma$-algebra on $C$. Therefore there exist $\mathcal{W}$-measurable bounded and continuous functions $F_{p, q}: C\left([0, T] ; \mathbb{R}^{d}\right) \rightarrow \mathbb{R}$ such that $\overline{\mathbb{E}}\left[\left(F_{p, q}(W)-\right.\right.$ $\left.\left.F_{q}(W)\right)^{2}\right] \rightarrow 0$ as $p \rightarrow \infty$. Along with Hölder's inequality we thus obtain

$$
\begin{aligned}
& \left|S_{1}(n, q)-\overline{\mathbb{E}}\left[g\left(W, \sqrt{n}\left(\bar{X}_{n}^{(q)}-X^{(q)}\right)\right) F_{p, q}(W)\right]\right| \\
& \leq\left(\overline{\mathbb{E}}\left[g\left(W, \sqrt{n}\left(\bar{X}_{n}^{(q)}-X^{(q)}\right)\right)^{2}\right]\right)^{1 / 2}\left(\overline{\mathbb{E}}\left[\left(F_{q}(W)-F_{p, q}(W)\right)^{2}\right]\right)^{1 / 2} \rightarrow 0
\end{aligned}
$$


as $p \rightarrow \infty$. Since $g$ is bounded, this convergence is uniform in $n \in \mathbb{N}$. Consequently we can exchange the limits and we obtain by (5.7) that

$$
\begin{aligned}
\lim _{n \rightarrow \infty} S_{1}(n, q) & =\lim _{n \rightarrow \infty} \lim _{p \rightarrow \infty} \overline{\mathbb{E}}\left[g\left(W, \sqrt{n}\left(\bar{X}_{n}^{(q)}-X^{(q)}\right)\right) F_{p, q}(W)\right] \\
& =\lim _{p \rightarrow \infty} \lim _{n \rightarrow \infty} \overline{\mathbb{E}}\left[g\left(W, \sqrt{n}\left(\bar{X}_{n}^{(q)}-X^{(q)}\right)\right) F_{p, q}(W)\right] \\
& =\lim _{p \rightarrow \infty} \overline{\mathbb{E}}\left[g\left(W, U^{(q)}\right) F_{p, q}(W)\right] \\
& =\overline{\mathbb{E}}\left[g\left(W, U^{(q)}\right) \mathbb{1}_{A_{q}}(W)\right]
\end{aligned}
$$

for every fixed $q \geq q_{0}$. For the latter step one can proceed as in (5.11). On the other hand, by Lemma 5.3 the integrand of $S_{2}(n, q)$ converges $\mathbb{P}$-almost surely to 0 as $n \rightarrow \infty$, so that dominated convergence yields $S_{2}(n, q) \rightarrow 0$ as $n \rightarrow \infty$ for every fixed $q \geq q_{0}$. Thus, using the dominated convergence theorem once again, we obtain

$$
\lim _{n \rightarrow \infty} \overline{\mathbb{E}}\left[g\left(W, U_{n}\right)\right]=\sum_{q=q_{0}}^{\infty} \overline{\mathbb{E}}\left[g\left(W, U^{(q)}\right) \mathbb{1}_{A_{q}}\right] .
$$

Since Lemma 5.4 implies

$$
\sum_{q=q_{0}}^{\infty} \overline{\mathbb{E}}\left[g\left(W, U^{(q)}\right) \mathbb{1}_{A_{q}}\right]=\sum_{q=q_{0}}^{\infty} \overline{\mathbb{E}}\left[g(W, U) \mathbb{1}_{A_{q}}\right]=\overline{\mathbb{E}}[g(W, U)],
$$

we reach (5.10).

\subsection{Proof of Lemma 5.3}

We first show that there are $\Omega_{1}, \Omega_{2} \in \mathcal{F}$ with $\mathbb{P}\left(\Omega_{1}\right)=\mathbb{P}\left(\Omega_{2}\right)=1$, such that for every $\omega \in \Omega_{1} \cap \Omega_{2} \cap\left\{\tau_{q}=\infty\right\}$ there are $\varepsilon(\omega)>0$ and $n_{0}(\omega) \in \mathbb{N}$ satisfying

$$
\begin{gathered}
\inf _{t \in[0, T]} \operatorname{dist}\left(X(t, \omega), \partial D_{q}\right)=\inf _{t \in[0, T]} \operatorname{dist}\left(X^{(q)}(t, \omega), \partial D_{q}\right)>\varepsilon(\omega), \\
\sup _{t \in[0, T]}\left|\bar{X}_{n}(t, \omega)-X(t, \omega)\right|+\sup _{t \in[0, T]}\left|\bar{X}_{n}^{(q)}(t, \omega)-X^{(q)}(t, \omega)\right|<\varepsilon(\omega)
\end{gathered}
$$

for all $n \geq n_{0}(\omega)$, where $\operatorname{dist}\left(x, \partial D_{q}\right)=\inf \left\{|x-y|: y \in \partial D_{q}\right\}$. To show (5.12) we may pick by Lemma 5.2 some $\Omega_{1} \in \mathcal{F}$ with $P\left(\Omega_{1}\right)=1$ such that $X\left(. \wedge \tau_{q}(\omega), \omega\right)=$ $X^{(q)}\left(. \wedge \tau_{q}(\omega), \omega\right)$ for all $\omega \in \Omega_{1}$. Thus we have $X(., \omega)=X^{(q)}(., \omega)$ for all $\omega \in \Omega_{1} \cap$ $\left\{\tau_{q}=\infty\right\}$. Then, since $D_{q}$ is an open set, there exists for every $\omega \in \Omega_{1} \cap\left\{\tau_{q}=\infty\right\}$ 
an $\varepsilon(\omega)>0$ satisfying (5.12). We next show (5.13). From [5] it follows that there exists a set $\Omega_{2} \in \mathcal{F}$ with $P\left(\Omega_{2}\right)=1$ such that

$$
\lim _{n \rightarrow \infty} \sup _{t \in[0, T]}\left|\bar{X}_{n}(t, \omega)-X(t, \omega)\right|=\lim _{n \rightarrow \infty} \sup _{t \in[0, T]}\left|\bar{X}_{n}^{(q)}(t, \omega)-X^{(q)}(t, \omega)\right|=0
$$

for all $\omega \in \Omega_{2}$. Thus for all $\omega \in \Omega_{2}$ there exists an $n_{0}(\omega) \in \mathbb{N}$ such that (5.13) holds.

From (5.12) and (5.13) it now follows that for all $\omega \in \Omega_{1} \cap \Omega_{2} \cap\left\{\tau_{q}=\infty\right\}$ there exists an $n_{0}(\omega) \in \mathbb{N}$ such that

$$
\bar{X}_{n}(t, \omega) \in D_{q}, \quad \bar{X}_{n}^{(q)}(t, \omega) \in D_{q}
$$

for all $t \in[0, T]$ and all $n \geq n_{0}(\omega)$.

To complete the proof of Lemma 5.3, we point to the following representation of the continuous time Euler scheme $\bar{X}_{n}$ :

$$
\bar{X}_{n}(t)=\bar{X}_{n}(k / n)+\sum_{j=0}^{m} f_{j}\left(\bar{X}_{n}(k / n)\right)\left(W_{j}(t)-W_{j}(k / n)\right)
$$

for $t \in(k / n,(k+1) / n]$; an analogous representation holds for $\bar{X}_{n}^{(q)}$. Thus, since $P\left(\Omega_{1} \cap \Omega_{2}\right)=1$, it obviously remains to show that for every $\omega \in \Omega_{1} \cap \Omega_{2} \cap\left\{\tau_{q}=\infty\right\}$ and all $n \geq n_{0}(\omega)$ we have

$$
\bar{X}_{n}(k / n, \omega)=\bar{X}_{n}^{(q)}(k / n, \omega), \quad k=0, \ldots, N_{n}(T) .
$$

However, due to $\bar{X}_{n}(0, \omega)=x_{0}=\bar{X}_{n}^{(q)}(0, \omega),(5.14)$, and $f_{j, q} \equiv f_{j}$ on $\bar{D}_{q}$, this follows straightforwardly by induction.

\subsection{Proof of Lemma 5.4}

For $1 \leq i \leq d$ set $Z_{i}=U_{i}-U_{i}^{(q)}$. Using the SDEs for $U$ and $U^{(q)}$, we obtain by Lemma A. 4 in the Appendix A that

$$
\begin{aligned}
& Z_{i}\left(t \wedge \tau_{q}\right) \\
& =\sum_{j=0}^{m} \int_{0}^{t} \nabla f_{i j}(X(s))^{\prime} Z(s) \mathbb{1}_{\left\{s \leq \tau_{q}\right\}} d W_{j}(t) \\
& \quad+\sum_{j=0}^{m} \int_{0}^{t}\left[\nabla f_{i j}(X(s))^{\prime}-\nabla f_{i j, q}\left(X^{(q)}(s)\right)^{\prime}\right] U^{(q)}(s) \mathbb{1}_{\left\{s \leq \tau_{q}\right\}} d W_{j}(s) \\
& -\frac{1}{\sqrt{2}} \sum_{j, l=1}^{m} \int_{0}^{t}\left[\nabla f_{i j}(X(s))^{\prime} f_{l}(X(s))\right. \\
& \left.\left.-\nabla f_{i j, q}\left(X^{(q)}(s)\right)^{\prime} f_{l, q}\left(X^{(q)}\right)(s)\right)\right] \mathbb{1}_{\left\{s \leq \tau_{q}\right\}} d B_{l j}(t) .
\end{aligned}
$$


Since $f_{i j, q}(x)=f_{i j}(x)$ for $x \in \bar{D}_{q}$, and $X\left(. \wedge \tau_{q}\right)=X^{(q)}\left(. \wedge \tau_{q}\right)$ by Lemma 5.2, the latter two summands vanish. So we have

$$
Z_{i}\left(t \wedge \tau_{q}\right)=\sum_{j=0}^{m} \int_{0}^{t} \nabla f_{i j}(X(s))^{\prime} Z\left(s \wedge \tau_{q}\right) \mathbb{1}_{\left\{s \leq \tau_{q}\right\}} d W_{j}(t),
$$

or equivalently,

$$
Z\left(t \wedge \tau_{q}\right)=\int_{0}^{t} d S_{q}(s) Z\left(s \wedge \tau_{q}\right)
$$

with $S_{q}=S \mathbb{1}_{\left\{s \leq \tau_{q}\right\}}$ and $S$ defined as in (5.2). Thus $Z\left(. \wedge \tau_{q}\right)$ satisfies a linear SDE with zero initial value, and Lemma A.3 in the Appendix A now implies $Z\left(. \wedge \tau_{q}\right) \equiv 0$ $\overline{\mathbb{P}}$-almost surely.

\section{A. Auxiliaries}

Here we give some auxiliaries. We start with the definition of strong solutions of SDEs driven by Brownian motion.

Definition A.1. Let $(\Omega, \mathcal{F}, \mathbb{P})$ be a probability space, and $W=(W(t): t \in[0, T])$ be an $m$-dimensional Brownian motion on $(\Omega, \mathcal{F}, \mathbb{P})$. Moreover, let $\mathcal{F}_{t}^{0}$ be the $\sigma$-algebra generated by $W$ up to time $t$, and $\left(\mathcal{F}_{t}\right)$ be the usual augmentation of the filtration $\left(\mathcal{F}_{t}^{0}\right)$. Finally, let $b, \sigma_{1}, \ldots, \sigma_{m}: \mathbb{R}^{d} \rightarrow \mathbb{R}^{d}$ be measurable functions. Then, a $d$-dimensional $\left(\mathcal{F}_{t}\right)$-adapted continuous process $X=(X(t): t \in[0, T])$ is called strong solution of the SDE

$$
d X(t)=b(X(t)) d t+\sum_{j=1}^{m} \sigma_{j}(X(t)) d W_{j}(t), \quad X(0)=x_{0},
$$

with respect to $\{(\Omega, \mathcal{F}, \mathbb{P}) ; W\}$ if for every $t \in[0, T]$,

$$
\int_{0}^{t}\left(|b(X(s))|+\sum_{j=1}^{m}\left|\sigma_{j}(X(s))\right|^{2}\right) d s<\infty \quad \mathbb{P} \text {-a.s. }
$$

and $\mathbb{P}$-almost surely

$$
X(t)=x_{0}+\int_{0}^{t} b(X(s)) d s+\sum_{j=1}^{m} \int_{0}^{t} \sigma_{j}(X(s)) d W_{j}(s), \quad t \in[0, T] .
$$

The solution is said to be (strongly) unique if any two strong solutions with respect to $\{(\Omega, \mathcal{F}, \mathbb{P}) ; W\}$ are $\mathbb{P}$-indistinguishable.

In this article, we also deal with affine SDEs driven by certain semi-martingales: 
Definition A.2. Let $(\Omega, \mathcal{F}, \mathbb{P})$ be a probability space, and $S=(S(t): t \in[0, T])$ and $H=(H(t): t \in[0, T])$ be $\mathbb{R}^{d, d}$ - resp. $\mathbb{R}^{d \text {-valued continuous semi-martingales }}$ on $(\Omega, \mathcal{F}, \mathbb{P})$. Moreover, let $\mathcal{F}_{t}^{0}$ be the $\sigma$-algebra generated by $S$ and $H$ up to time $t$, and $\left(\mathcal{F}_{t}\right)$ be the usual augmentation of the filtration $\left(\mathcal{F}_{t}^{0}\right)$. Then, a $d$-dimensional $\left(\mathcal{F}_{t}\right)$-adapted continuous process $U=(U(t): t \in[0, T])$ is called solution of the SDE

$$
d U(t)=d H(t)+d S(t) U(t), \quad U(0)=H(0),
$$

with respect to $\{(\Omega, \mathcal{F}, \mathbb{P}) ; S ; H\}$ if for every $t \in[0, T]$,

$$
\int_{0}^{t}|U(s)|^{2} d s<\infty \quad \mathbb{P} \text {-a.s. }
$$

and $\mathbb{P}$-almost surely

$$
U(t)=H(t)+\int_{0}^{t} d S(s) U(s), \quad t \in[0, T] .
$$

The solution is said to be unique if any two solutions w.r.t. $\{(\Omega, \mathcal{F}, \mathbb{P}) ; S ; H\}$ are $\mathbb{P}$ indistinguishable.

Note that the order of $d S(s) U(s)$ in (A.3) and (A.4) is not a mistake. Alternatively we could have written $\left(U(s)^{\prime} d S(s)^{\prime}\right)^{\prime}$. The same applies to (A.5) below. SDE (A.3) always has a unique solution which can be represented as follows (this result can be found e.g. in a more general setting in [7]):

Lemma A.3. In the setting of Definition A.2, SDE (A.3) has a unique solution with respect to $\{(\Omega, \mathcal{F}, \mathbb{P}) ; S ; H\}$. This solution is given by

$$
U(t)=\Phi(t) H(0)+\Phi(t) \int_{0}^{t} \Phi(s)^{-1} d G(s), \quad t \in[0, T],
$$

where $\Phi=(\Phi(t): t \in[0, T])$ is the unique solution of the $\mathbb{R}^{d, d}$-valued linear SDE

$$
d \Phi(t)=d S(t) \Phi(t), \quad \Phi(0)=\mathbb{I}_{d}
$$

and

$$
G=H-\langle S, H\rangle \text {. }
$$

Here $\Phi(s)^{-1}$ denotes the inverse of the matrix $\Phi(s)$, and $\langle S, H\rangle=\left(\langle S, H\rangle_{i}\right)_{i=1}^{d}$ with $\langle S, H\rangle_{i}=\sum_{j=1}^{d}\left\langle S_{i j}, H_{j}\right\rangle$.

In Subsection 5.5 we need the following stopping rule for Itô-integrals. For its proof see e.g. Proposition III.2.10 and the remark on page 147 in [12]. 
Lemma A.4. Let $(Y(t): t \in[0, T])$ be a real-valued $\left(\mathcal{F}_{t}\right)$-progressively measurable stochastic process on any filtered probability space $\left(\Omega, \mathcal{F},\left(\mathcal{F}_{t}\right), \mathbb{P}\right)$ with

$$
\int_{0}^{T}|Y(t)|^{2} d t<\infty \quad \mathbb{P} \text {-a.s. }
$$

and $(W(t): t \in[0, T])$ be a one-dimensional $\left(\mathcal{F}_{t}\right)$-Brownian motion. Let moreover $\tau$ be an $\left(\mathcal{F}_{t}\right)$-stopping time. Then it holds $\mathbb{P}$-almost surely

$$
\int_{0}^{t \wedge \tau} Y(s) d W(s)=\int_{0}^{t} Y(s) \mathbb{1}_{\{s \leq \tau\}} d W(s), \quad t \in[0, T] .
$$

\section{References}

1. D.J. Aldous And G.K. Eagleson On mixing and stability of limit theorems. Annals of Probability 6, p. 325-331. (1978)

2. L. Arnold, G. Bleckert And K.R. SchenK-Hoppé The stochastic Brusselator: Parametric noise destroys Hopf bifurcation. In: H. Crauel (ed.) et al. Stochastic Dynamics. Springer, New York, p. 71-92. (1999)

3. J. Cox, J. Ingersoll AND S. Ross A theory of the term structure of interest rates. Econometrica 53, p. 385-408. (1985)

4. T. Fischer, A. MAY AND B. WALther Simulation of the yield curve: checking a CoxIngersoll-Ross model. Working Paper (2002)

5. I. GyÖNGY A note on Euler's approximations. Potential Analysis 8, p. 205-216. (1998)

6. S.L. HESTON A closed-form solution for options with stochastic volatility with applications to bond and currency options. The Review of Financial Studies 6, p. 327-343. (1993)

7. J. JACOD Equations différentielles stochastiques linéaires: La méthode de variation des constantes. Séminaire de probabilités XVI - Lecture Notes in Mathematics 920, p. 442446. (1982)

8. J. Jacod And P. Protter Asymptotic error distributions for the Euler method for stochastic differential equations. Annals of Probability 26, p. 267-307. (1998)

9. J. Jacod And A.N. Shiryaev Limit Theorems for Stochastic Processes. Springer, Berlin, 2nd ed. (2003)

10. A. Jentzen, P.E. Kloeden And A. Neuenkirch Pathwise approximation of stochastic differential equations on domains: Higher order convergence rates without global Lipschitz coefficients. Numerische Mathematik 112, p. 41-64. (2009)

11. O. Kallenberg Foundation of Modern Probability. Springer, Berlin. (1997)

12. I. Karatzas And S.E. Shreve Brownian Motion and Stochastic Calculus. Springer, New York, 2nd edition. (1991)

13. P.E. Kloeden And E. Platen Numerical Solution of Stochastic Differential Equations. Springer, Berlin, 3rd edition. (1999) 
14. T.G. KURTZ AND P. PROTTER Wong-Zakai corrections, random evolutions, and simulation schemes for SDE's. In: E. Mayer-Wolf (ed.) et al. Stochastic Analysis, p. 331-346. (1991)

15. D. LÉPINGLE Euler scheme for reflected stochastic differential equations. Mathematics and Computers in Simulation 38, p. 119-126. (1995)

16. G.N. MiLsteIn Numerical Integration of Stochastic Differential Equations. Kluwer, Doordrecht. (1995)

17. R. Pettersson Approximations for stochastic differential equation with reflecting convex boundaries. Stochastic Processes and their Applications 59, p. 295-308. (1995)

18. D. Pollard Convergence of Stochastic Processes. Springer, New York. (1984)

19. M. Scheutzow Periodic behavior of the Stochastic Brusselator in the mean field limit. Probability Theory and Related Fields 72, p. 425-462. (1986)

20. H. ZÄHLE Weak approximation of SDEs by discrete-time processes. Journal of Applied Mathematics and Stochastic Analysis 2008, 14 pages. (2008)

21. H. ZÄHLE Approximation of SDEs by population-size-dependent Galton-Watson processes. Stochastic Analysis and Applications (2009), to appear

\section{Author information}

Andreas Neuenkirch, Technische Universität Dortmund, Fakultät für Mathematik, Vogelpothsweg 87, D-44227 Dortmund, Germany.

Email: andreas.neuenkirch@math.tu-dortmund.de

Henryk Zähle, Technische Universität Dortmund, Fakultät für Mathematik, Vogelpothsweg 87, D-44227 Dortmund, Germany.

Email: henryk.zaehle@math.tu-dortmund.de 


\section{Preprints ab 2008/08}

2010-09

2009-09

2009-08

2009-07

2009-06

2009-05

2009-04

2009-02

2009-01

$2008-25$

$2008-24$

$2008-23$

$2008-22$

2008-21
Andreas Neuenkirch and Henryk Zähle

Asymptotic error distribution of the Euler method for SDEs with non-Lipschitz coefficients

Karl Friedrich Siburg, Pavel A. Stoimenov

Regression dependence

Wilfried Hazod

Continuous convolution hemigroups integrating a sub-multiplicative function

Sergio Conti and Ben Schweizer

On optimal metrics preventing mass transfer

Simon Castle, Norbert Peyerimhoff, Karl Friedrich Siburg

Billiards in ideal hyperbolic polygons

Ludwig Danzer

Quasiperiodic Tilings - Substitution Versus Inflation

Flavius Guiaş

Direct simulation of the infinitesimal dynamics of semi-discrete

approximations for convection-diffusion-reaction problems

Franz Kalhoff and Victor Pambuccian

Existential definability of parallelism in terms of betweenness

in Archimedean ordered affine geometry

Fulvia Buzzi, Michael Lenzinger and Ben Schweizer

Interface conditions for degenerate two-phase flow equations

in one space dimension

Henryk Zähle

Approximation of SDEs by population-size-dependent

Galton-Watson processes

Winfried Hazod

Mehler semigroups, Ornstein-Uhlenbeck processes and background driving Lévy processes on locally compact groups and on hypergroups

Karl Friedrich Siburg, Pavel A. Stoimenov

Symmetry of functions and exchangeability of random variables

Ina Kirsten Voigt

Voronoi Cells of Discrete Point Sets

Michael Lenzinger and Ben Schweizer

Effective reaction rates of a thin catalyst layer

Michael Voit

Bessel convolutions on matrix cones: Algebraic properties and random walks 
Margit Rösler and Michael Voit

Limit theorems for radial random walks on $p \times q$-matrices as $p$ tends to infinity

2008-19

Michael Voit

Central Limit Theorems for Radial Random Walks on $p \times q$ Matrices for $p \rightarrow \infty$

2008-18 Michael Voit

Limit theorems for radial random walks on homogeneous spaces with growing dimensions

2008-17 Ansgar Steland and Henryk Zähle

Sampling inspection by variables: nonparametric setting

2008-16 Guy Bouchitté and Ben Schweizer

Homogenization of Maxwell's equations with split rings

2008-15 Wilfried Hazod

Multiple selfdecomposable laws on vector spaces and on groups:

The existence of background driving processes

2008-14 Wilfried Hazod

Mixing of generating functionals and applications to (semi-)stability of probabilities on groups

2008-13 Wilfried Hazod

Probability on Matrix-Cone Hypergroups: Limit Theorems and Structural Properties

2008-12 Michael Lenzinger and Ben Schweizer

Two-phase flow equations with outflow boundary conditions in the hydrophobic-hydrophilic case

2008-11 Karl Friedrich Siburg

Geometric proofs of the two-dimensional Borsuk-Ulam theorem

2008-10 Peter Becker-Kern, Wilfried Hazod

Mehler hemigroups and embedding of discrete skew convolution

2008-09 Karl Friedrich Siburg, Pavel A. Stoimenov

Gluing copulas

2008-08 Karl Friedrich Siburg, Pavel A. Stoimenov

A measure of mutual complete dependence 